\title{
A Study on performance enhancement of VLC systems using MIMO techniques
}

\author{
Dr.R.Venkateswari ${ }^{1}$, Fathima Suhana $\mathrm{M}^{2}$ and Divya $\mathrm{R}^{3}$ \\ \{rvi.ece@psgtech.ac.in ${ }^{1}, 1907$ n101@psgtech.ac.in², divshivanga@gmail.com ${ }^{3}$ \} \\ Dept.of ECE, PSG College of Technology Coimbatore, India ${ }^{1}$
}

\begin{abstract}
There is dramatic increase in wireless traffic due to the demand of high speed internet services such as M2M, Cloud based services, video conferences, online gaming, web browsing etc. This huge global demand of bandwidth find it complex to accomodate in the existing RF spectrum. So alternative wireless technologies that will ease this 'Spectrum crunch' need to be implemented as soon as possible. The technology must be able to provide ultra high data rates to end users. Visible Light Communication, which is a subset of Optical Wireless communi- cation technology have unregulated, unlicensed bandwidth.It can provide dual function of illumination and communication at high data rates.This paper gives a study on performance enhancement of MIMO VLC Systems.
\end{abstract}

Keywords: Optical wireless communication, VLC, MIMO- OFDM VLC, Precoding, Spatial diversity.

\section{Introduction}

Next generation wireless data services include scenarios like M2M communication, AR/VR,ultra-low and ultra-high reliable (URLLC)networks, massive connectivity of devices, higher energy efficiency etc. The existing RF spectrum is crowded which causes RF interference, that deteriorates the performance of wireless services. Multipath propagation reduces link availability in dense environments. So an alternative technology that is highly reliable and of low cost must be relied upon to reduce the RF spectrum congestion. One of the possible solutions is Optical Wireless Communication Technology that uses IR (Infrared), UV(Ultraviolet), visible light sub bands for communication. These techniques can pro- vide ultra-high bandwidth (order of THz), unlimited frequency reuse, physical security, unlicensed band etc. Moreover, it is not subject to electromagnetic interference. Visible Light Communication (VLC) system work in the visible band of 390-700 nm. Here, Light emitting diodes (LEDs) enable dual purpose of communication at high speeds and illumination. VLC can be applied in wireless local area, personal area, body area networks, indoor localization, heterogeneous networks, under- ground, underwater networks, areas sensitive to electromagnetic radiation etc. There are many challenges faced by VLC such asambient light sources, integration with existing technologies, interference between VLC devices, mobility issues such as handover, performance improvement etc.VLC was standardised by efforts such as JEITA (Japan Electronics and In- formation Technology Industries Asociation(JEITA CP1221, CP1222, CP1223) and IEEE (IEEE 802.15.7).In [1],MIMO VLC system proposed with narrow FOV photodiodes. In [7], transceiver system for MISO (Multi Input Single Output) VLC system with CSI (Channel State Information) is proposed to control 
interference. In [8], indoor data connectivity,co- channel interference and intersymbol interference issues are addressed.CFOV-ADR(Constrained Field Of View Angle Di- versity Receiver) and LS (Least Square) channel estimation with ML (Maximum Likelihood) equalizer is used.In [13], authors propose ZCT (Zadoff Chu matrix transform) precoding to improve performance of STBC (Space Time Block Codes) based MIMO OFDM VLC system.In [14], spatial crosstalk and temporal interference is mitigated by employing angle diversity receiver, ZF equalization and decision feedback equalization.In [18],complex modulation schemes for MIMO VLC are proposed and their performance is analyzed in terms of BER. In [22], the inter-cell interference in mitigated by ADR with SBC (Select Best Combining),EGC (Equal Gain Combining) and MRC (Maximum Ratio Combining).In [23], the performance of VLC system for 5G network us- ing Massive MIMO, MIMO OFDM etc is reviewed.In [25], FGIS (Fully Generalized Index Spatial) modulation scheme is proposed to increase spectral efficiency of optical MIMO OFDM system.In [27], spectral efficiency improvement for U- OFDM (unipolar OFDM) with adaptive transmission through realistic VLC links is proposed. In [29], the performance of MIMO modulation schemes is enhanced using imaging receiver with hemispherical and fish-eye lens.Here, the lens provide increased spatial resolution.In [31],the performance of precoding techniques in MIMO VLC systems is analyzed.In [32], authors propose an optical MIMO VLC system based on modified OFDM/OQAM inorder to mitigate IMI (Intrinsic Imaginary Interference).In [35],ZF-SIC (Zero Forcing Suc- cessive Interference Cancellation) receiver with SVD based index precoding is proposed for practical VLC deployments.In [40], authors propose a generalised LED index modulation scheme for MIMO-OFDM VLC system to improve spectral efficiency.In [41], capacity of MIMO-OFDM VLC systems enhanced by OCT (Orthogonal Circulant matrix Transform) precoding and SVD (Singular Value Decomposition) based adaptive loading optimization is proposed.In [43], an extensive survey on research activities and advances in VLC under MU- MIMO (Multi User MIMO), OFDM is provided. In [44], an adaptive transmission for MIMO VLC system by power and bit allocation to enhance capacity is proposed.In [49], realistic indoor visible light channels are proposed in MIMO transmission scenario.Non sequential ray tracing approach for CIR (Channel Impulse Response) is used.In [50], NHS-OFDM (Non Hermitian Symmetry) scheme is investigated for MIMO- VLC systems with imaging and non-imaging receiver.

\section{VLC System}

VLC technology is based on Solid State Lighting (SSL) ie generating light through electroluminescence. SSL has lower power consumption, highly tolerant to humidity, mercury-free, driver circuit convert voltage to current signals in order to excite LEDs for dual functionality. The current input to LEDs is controlled by LED driver,so that emitted light is at high frequency, unperceivable by human eyes. Spatially distributed clusters of LEDs are used for large indoor coverage.LEDs are preferred over Lased diode (LD) in VLC since it serves purposes of illumination, data communication, sensing, local- ization etc. Mostly OWC technologies share common link con- figuration. The four common link configurations in literature are Directed LOS, Non directed LOS, Non directed NLOS and Tracked. The channel model used in VLC is a linear, time- invariant, finite impulse response, memoryless system. The mathematical representation of channel impulse response is considered as a transmission matrix.The emitter beam angle and receiver Field of view determine the 
transmission chan- nel. Equalization techniques helps to recover symbols during interference due to noise or unwanted sources. The optical receiver is composed of phototdetector, amplifier, limiting amplifier and so on. The detection scheme used here is called IM/DD (Intensity Modulation/Direct Detection).

$i(m+1) A$

Compact in size, fast switching of data, longer life expectancy etc. The type of modulation used is IM (Intensity Modulation),

$H_{i}=2 \pi_{d}^{2} \cos ^{m} \varphi_{i} T_{s} g\left(\psi_{i}\right) \cos \psi_{i}, \quad 0 \leq \psi_{i} \leq \phi_{c}(1)$

since phase/frequency modulation of incoherent waves is difficult.Every LED act as wireless access points (APs) and can support multiple UE (User Equipment) devices. Secure indoor data links are assured by eliminating eavesdropping and interception since light do not propagate through opaque ob- jects. Moreover VLC enables safe transmission in areas where

RF Communication is prohibited such as hospitals, aviation,

A is the area of photodetector, $d_{i}$ is Euclidean distance between $A P_{i}$ and $\mathrm{UE}, \varphi_{i}$ is the angle of radiance with respect to normal to transmitter for $A P_{i}, \psi_{i}$ is angle of incidence with respect to normal to receiver. $T_{s}$ is gain of optical filter, $\phi_{c}$ is FOV(Field of View). The optical path loss which is the ratio of luminous flux at receiver to that at transmitter is given by:

Mines, petrochemical,nuclear power units etc. VLC can be successfully commercialized in the coming years. Fig shows

$D 2 \int \theta \max 2 \pi g(\theta) \sin \theta d \theta(2)$

A typical VLC Sytem Block Diagram.It consists of backbone network,transmitter part,indoor channel and receiver part. VLC Here $A_{r}$ is the area of Receiver, D is distance between transmitter and receiver.The received optical power in direct LOS link is given by:

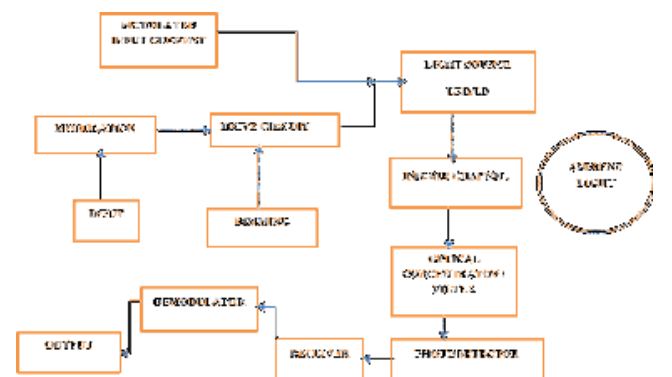

Fig. 1. VLC System Block Diagram

$P_{R} O=S_{R}(\lambda) R_{f}(\lambda) d \lambda \quad(3)$

Transmitter circuit consists of signal generator, RLL (Run Length Limited) coder, Channel coder for FEC (Forward Error Correction), Modulator, LED Driver and LEDs.LED arrays are used here which contains more than one LED.LEDs are assumed as point sources with Lambertian emission patterns (the surface gives same illuminance when observed through Here $R_{f}(\lambda)$ is spectral response of optical filter.The received power depends on incident angle, irradiance angle, transmitter-receiver distance. The parameters will change on user ori- entation and movement.The modulation is done so that the signal becomes adapted for channel transmission, to provide dimming control, flicker mitigation, so that dual functioning of VLC is balanced, to switch LEDs to desired frequencies using drivers and so on. The modulation methods used in VLC are OOK,VPPM, OFDM, CSK (Colour Shift Keying). CSK is used for multiple optical sources with different frequencies or colours. The 
optical variants of OFDM are DC-biased OFDM, Asymmetrically Clipped OFDM etc for obtaining real valued signals. The SNR of VLC system is proportional to square of received optical power ie $\left(R H(0) P_{r}\right)^{2}$

Different angles, placed on vertices of ceiling of a room. The Here $H(0)$ is DC gain of channel, $\mathrm{R}$ is responsivity of $\mathrm{PD},{ }^{2}$ is noise variance. There are many limitations in designing

a VLC system. They are performance degradation due to blocking/shadowing,non-linear power characteristics of LED, ISI, low modulation bandwidth of LED, Communication with dimming, uplink design, compatibility with existing networks, coverage, link span etc. An optical bidirectional beacon can be used to increase coverage area and link availability during shadowing/blocking. Distributed light sources and angle diver- sity receivers can also be used to avoid blocking.. The CIR can be calculated as:

$j=1$

$h(t)=\Sigma^{N} r \quad P_{j} \delta\left(t-\tau_{j}\right)(5)$

ray, $\delta(t)$ is Dirac delta function, $N_{r}$ is number of rays received $P_{j}$ is optical power of $\mathrm{j}$-th ray, $\tau_{j}$ is propagation delay of $\mathrm{j}$-th by detector.

\section{MIMO OFDM VLC Model}

The modulation bandwidth of LEDs is very limited ie only several megahertz can be used.The bandwidth limitation of LEDs can be reduced by MIMO-VLC system.In MIMO OFDM VLC system model, ther are $\mathrm{T}$ transmitters and $\mathrm{R}$ receivers. The data is converted into $\mathrm{S} / \mathrm{P}$ (serial to parallel) and mapped in frequency domain, such as QAM(Quadrature Amplitude Modulation) mapping.Here, spatial diversity increases throughput and Spatial multiplexing increases data rate.In MIMO VLC system, each lamp is composed of multiple LEDs with different inclination angles. The receiver contains PDs with different inclination angles.Multiple receiving elements aid for demultiplexing and decoding. The total number of LEDs is $N_{Q}$.

$$
N_{Q}=N \times Q(6)
$$

Here $\mathrm{N}$ is the number of lamps, $\mathrm{Q}$ is the number of LEDs. The $\times$ number of users in the indoor environment is $\mathrm{K}$ and number of photo detectors be $\mathrm{V}$. The performance of MIMO system depends on channel correlation.In Spatial Multiplexing, the lumnaires emit independent data streams.In [5], the author investigates imaging and non-imaging MIMO systems. In [20] structure of imaging and non-imaging MIMO systems are pro- vided.High data rates despite of limited modulation bandwidth of LEDs can be achieved by Spatial Multiplexing. In Non- imaging MIMO system, non-imaging lenses are used to collect transmitted intensity. The light from LEDs are collected at different intensities by optical concentrators. The MIMO channel matrix, $\mathrm{H}$ is of dimension $N_{R} \quad N_{T}$, where $N_{R}$ is the number of receiver elements and $N_{T}$ is the number of transmitter elements. The LEDs point downwards from the ceiling to transmit information. The received signal from multiple transmitters will suffer from Multi stream interference (MSI).In Optical spa- tial modulation, both data symbols provide information. These data symbols are transmitted orthogonally, thus eliminating MSI. The receiver front end must be able to decouple signals from multiple sources. Commonly used receiver front end for MIMO systems include prism array receivers, aperture- based receivers, $\mathrm{K}-\mathrm{FOV}$ (Field of view) receivers etc.ZF(Zero 
forcing) equalization help in decoupling signals at the receiver. Consider inddor VLC system with room dimensions $X x Y x Z$.MIMO receiver facing upwards at a height $\mathrm{T}$ from ground is placed.Here receiver elements are greater than trans- mitter elements.In Spatial multiplexing with ACO-OFDM, all odd subcarriers are loaded with data symbols and transmit- ted as independent data streams. The ACO-OFDM modulator converts complex bipolar data symbols into real non negative symbols. The signals are modulated onto light intensity by LED luminaire.

\section{Precoding for MIMO systems}

MIMO techniques can utilize available bandwidth effec- tively. The throughput of MIMO VLC system can be improved by precoding techniques such as Zero Forcing (ZF) precoding, ZF precoding is a prominent linear precoding scheme that achieves full spatial multiplexing gain and multiuser diversity gain. Both magnitude and phase information is weighted such that distance is normalized.The interference terms are forced to zero to eliminate multi user interference. Fig 1 shows MIMO

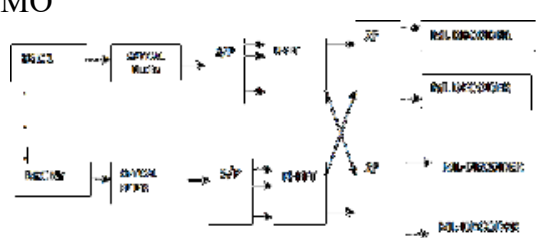

Fig. 2. MIMO Receiver with Spatial multiplexing

Receiver with Spatial Multiplexing. The received signals are converted from time domain to frequency domain by demod- ulator.Demultiplexing includes ZF-equalization followed by ML-decoding. The input data is multiplexed into $M$ parallel transmit beams, each of which intensity-modulates the light source.In [19], authors propose a spatial dimming scheme for MU-MIMO VLC system. The transmitted signal is precoded

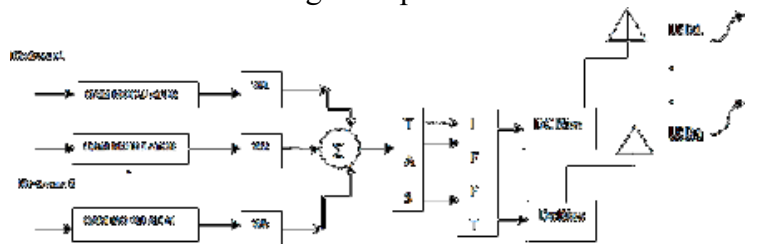

Fig. 3. MU MIMO OFDM VLC Transmitter

and DC-component is added.The dc-bias is added to ac component to achieve data communication without affecting primary illumination of LEDs.

- Channel Modelling of MIMO VLC system

Light from each LED array is received by seperate receivers with different intensities. The baseband channel model in dis- crete time for MIMO system is given by:

$y=H x+n$

Here $\mathrm{n}$ is the AWGN noise. Channel estimation coefficients between a pair of Tx and Rx need to be estimated to retrieve transmitted data. MIMO demultiplexing algorithms can 
successfully recover input signals. The commonly used such algorithms are ZF(Zero forcing), minimum mean square error (MMSE) etc.ZF is used widely due to its simplicity.

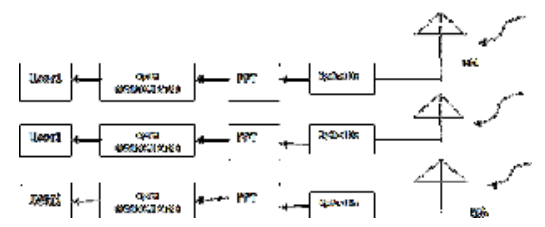

Fig. 4. MU MIMO OFDM VLC Receiver

\section{Simulation}

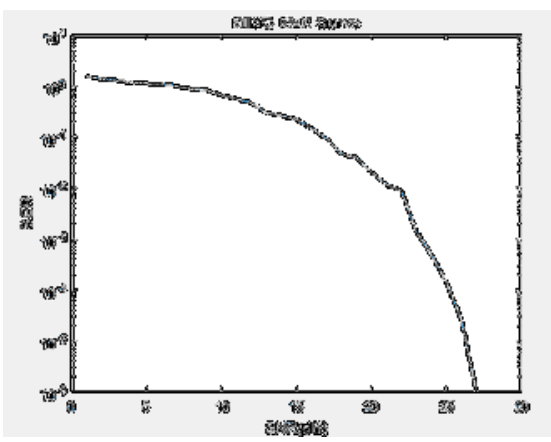

Fig. 5. BER vs SNR Curve

\section{A. SNR Analysis}

The signal is transmitted at a definite time slot.The position of transmitters on the effect of transmitted signal is consid- ered. LED is used as both illuminating and communicating device.The quality of communication can be expressed by SNR.Here the effect of multipath fading is ignored.The in- formation is present in the light wave.The electrical SNR can be expressed as: $\left(R P_{r}\right)^{2}$ 


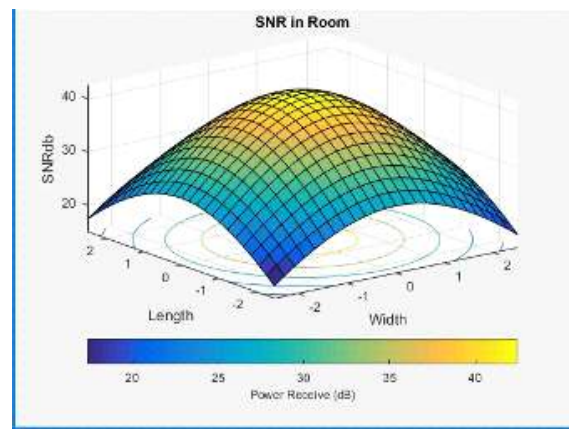

Fig. 6. SNR Distribution within room

\section{References}

[1] Ahmed Taher Ali et al 2021 IOP Conf. Ser.: Mater. Sci. Eng. 1152012009.

[2] C e'spedes, Ma'ximo M., Borja G. Guzma'n, and V'ictor P.G. Jime'nez 2021. "Lights and Shadows: A Comprehensive Survey on Cooperative and Precoding Schemes to Overcome LOS Blockage and Interference in Indoor VLC" Sensors 21, no. 3: 861. https://doi.org/10.3390/s21030861

[3] T. Ganjian, G. Baghersalimi and Z. Ghassemlooy, "Performance Eval- uation of a MIMO VLC System Employing Single Carrier Modulation with Frequency Domain Equalization," 2020 3rd West Asian Symposium on Optical and Millimeter-wave Wireless Communication (WASOWC), 2020, pp. 1-5, doi: 10.1109/WASOWC49739.2020.9410001.

[4] Di Zheng, Hongming Zhang, and Jian Song, Fellow, IEEE, "Spatial Multiplexing MIMO Visible Light Communications With Densely Dis- tributed LEDs and PDs", IEEE photonics journal,Vol 12 No.5,October 2020.

[5] C. Chen, I. Tavakkolnia, M. D. Soltani, M. Safari and H. Haas, "Hybrid multiplexing in OFDMbased VLC systems," 2020 IEEE Wireless Communications and Networking Conference (WCNC), 2020, pp. 1-6, doi: 10.1109/WCNC45663.2020.9120825.

[6] C. Chen et al., "User-Centric MIMO Techniques for Indoor Visible Light Communication Systems," in IEEE Systems Journal, vol. 14, no. 3, pp. 3202-3213, Sept. 2020, doi: 10.1109/JSYST.2019.2961696.

[7] J. Z. Zhang, K. Ke and J. Ren, "Multiuser Robust Transceiver Design for Indoor VLC with Noisy Channel State Information," 2020 IEEE International Conference on Progress in Informatics and Computing (PIC), 2020, pp. 289-293, doi: 10.1109/PIC50277.2020.9350831.

[8] M. Hosney, H. A. I. Selmy, A. Srivastava and K. M. F. Elsayed, "In- terference Mitigation Using Angular Diversity Receiver With Efficient Channel Estimation in MIMO VLC," in IEEE Access, vol. 8, pp. 54060- 54073, 2020, doi: 10.1109/ACCESS.2020.2981137.

[9] Jingyuan Feng, Chunyong Yang, Jin Hou, Hao Long, Shaop- ing Chen, Performance enhancement for indoor visible light communication system with an improved inter-symbol interfer- ence model using optimized hemispherical optical-angle-diversity- receivers, Optics Communications, Volume 4018,https://doi.org/10.1016/j.optcom.2019.124488.

[10] Ibrahim A. Elewah, Faezah Jasman, Shashiong Ng, "Performance en-Author AA. Title of book. Edition [if not first]. Place of publication: Publisher; Year of publication. Pagination.

[11] Y. Zhai, H. Chi, J. Tong and J. Xi, "Capacity Maximized Linear Precoder Design for SpatialMultiplexing MIMO VLC Systems," in IEEE Access, vol. 8, pp. 63901-63909, 2020, doi: 10.1109/ACCESS.2020.2983517. 
[12] [12]H. G. Olanrewaju, J. Thompson and W. O. Popoola, "Pairwise Coding for MIMO-OFDM Visible Light Communication," in IEEE Transactions on Wireless Communications, vol. 19, no. 2, pp. 1210-1220, Feb. 2020, doi: 10.1109/TWC.2019.2951666.

[13] [13]Xinyue Guo, Chuxian Wang, Wei Wang, "Experimental Demon- stration of Zadoff-Chu Matrix Transform Precoding for MIMO- OFDM Visible Light Communications", Advances in Condensed Matter Physics, vol. 2020, Article ID 4343582, 7 pages, 2020. https://doi.org/10.1155/2020/4343582

[14] F. Dong, R. Singh and D. O’Brien, "Adaptive MIMO-VLC Sys- tem for High Data Rate Communications," 2020 IEEE Globe- com Workshops (GC Wkshps, 2020, pp. 1-6, doi: 10.1109/GCWk- shps50303.2020.9367537.

[15] Mengru Chen, Huimin Lu, Danyang Chen, Jianli Jin, Jianping Wang, An efficient MIMOOFDM VLC system of combining space time block coding with orthogonal circulant matrix transform pre- coding, Optics Communications, Volume 473,2020,125993, ISSN 00304018,https://doi.org/10.1016/j.optcom.2020.125993.

[16] Lili Hao, Dongyi Wang, Wenyong Cheng, Jing Li, Anfan Ma, Perfor- mance enhancement of ACO-OFDM-based VLC systems using a hybrid autoencoder scheme,Optics Communications, Volume 442,2019, Pages 110-116,ISSN 00304018,https://doi.org/10.1016/j.optcom.2019.03.013.

[17] A. Kumar and S. K. Ghorai, "Performance of MIMO-VLC System for Different Radiation Patterns of LED in Indoor Optical wireless Com- munication System," 2019 IEEE International Conference on Advanced Networks and Telecommunications Systems (ANTS), 2019, pp. 1-5, doi: 10.1109/ANTS47819.2019.9118062.

[18] K. V. S. S. Sushanth and A. Chockalingam, "Multiple-LED Complex Modulation Schemes for Indoor MIMO VLC Systems," ICC 2019 - 2019 IEEE International Conference on Communications (ICC), 2019, pp. 1-6, doi: 10.1109/ICC.2019.8761132.

[19] Jesuthasan, Fabian Rohitkumar, Hardik Shah, Purav Tres- tian, Ramona. (2019). Implementation and Performance Evalu- ation of a MIMO-VLC System for Data Transmissions. 1-6. 10.1109/BMSB47279.2019.8971932.

[20] A. Khalid, H. M. Asif, S. Mumtaz, S. Al Otaibi and K. Konstantin, "Design of MIMO-Visible Light Communication Transceiver Using Maximum Rank Distance Codes," in IEEE Access, vol. 7, pp. 89128- 89140, 2019, doi: 10.1109/ACCESS.2019.2924202.

[21] U. Thummaluri, A. Kumar and L. Natarajan, "MIMO Codes for Uniform Illumination Across Space and Time in VLC With Dimming Control," in IEEE Photonics Journal, vol. 11, no. 3, pp. 121, June 2019, Art no. 7903721, doi: 10.1109/JPHOT.2019.2918063.

[22] P. P. J a'tiva, C. A. Azurdia-Meza, M. R. Cañizares, S. Ce'spedes and S. Montejo-Sa'nchez, "Performance Enhancement of VLC-Based Systems Using Diversity Combining Schemes in the Receiver," 2019 IEEE Latin- American Conference on Communications (LATINCOM), 2019, pp. 1-6, doi: 10.1109/LATINCOM48065.2019.8937854.

[23] Vishwaraj and L. Ali, "Hybrid MIMO-OFDM System for 5G Network Using VLC-A Review," 2019 IEEE International Conference on Electri- cal, Computer and Communication Technologies (ICECCT), 2019, pp. 1-5, doi: 10.1109/ICECCT.2019.8869299.

[24] S. Idris, U. Mohammed, J. Sanusi and S. Thomas, "Visible Light Communication: A potential 5G and beyond Communica- tion Technology," 2019 15th International Conference on Electron- ics, Computer and Computation (ICECCO), 2019, pp. 1-6, doi: 10.1109/ICECCO48375.2019.9043201.

[25] H. S. Hussein and M. Hagag, "Optical MIMO-OFDM With Fully Generalized Index-Spatial LED Modulation," in IEEE Communi- cations Letters, vol. 23, no. 9, pp. 1556-1559, Sept. 2019, doi: 10.1109/LCOMM.2019.2926457.

[26] Palacios, Pablo Azurdia-Meza, Cesar Roman Ca ñizares, Milton Ce'spedes, Sandra Montejo Sa'nchez, Samuel. (2019). Performance En- hancement of VLC-Based Systems Using Diversity Combining Schemes in the Receiver. 10.1109/LATINCOM48065.2019.8937854. 
[27] M. Al-Nahhal, E. Basar and M. Uysal, "Adaptive Unipolar MIMO- OFDM for Visible Light Communications," 2019 European Conference on Networks and Communications (EuCNC), 2019, pp. 73-77, doi: 10.1109/EuCNC.2019.8801994.

[28] A. Chaaban, Z. Rezki and M. Alouini, "Capacity Bounds and High- SNR Capacity of MIMO Intensity-Modulation Optical Channels," in IEEE Transactions on Wireless Communications, vol. 17, no. 5, pp. 3003-3017, May 2018, doi: 10.1109/TWC.2018.2805726.

[29] A. K. Gupta and A. Chockalingam, "Performance of MIMO Modulation Schemes With Imaging Receivers in Visible Light Communication," in Journal of Lightwave Technology, vol. 36, no. 10, pp. 1912-1927, 15 May15, 2018, doi: 10.1109/JLT.2018.2795698.

[30] A. Kumar and S. K. Ghorai, "BER performance analysis of indoor MIMO-VLC system for multipath reflection," 2018 Technologies for Smart-City Energy Security and Power (ICSESP), 2018, pp. 1-5, doi: 10.1109/ICSESP.2018.8376688.

[31] V. Arthi, S. P. Chakkravarthy and R. Ramya, "Performance Analysis of Precoding Techniques for MIMO VLC Systems," 2018 InternationalConference on Smart Systems and Inventive Technology (ICSSIT), 2018, pp. 434-437, doi: 10.1109/ICSSIT.2018.8748768.

[32] B. Lin, X. Tang, Z. Ghassemlooy, C. Lin, Z. Zhou and H. Zhang, "OFDM/OQAM for MIMO Visible Light Communications," 2018 Asia Communications and Photonics Conference (ACP), 2018, pp. 1-3, doi: 10.1109/ACP.2018.8596000.

[33] H. Yang, C. Chen, W. Zhong and A. Alphones, "Joint Precoder and Equalizer Design for MultiUser Multi-Cell MIMO VLC Systems," in IEEE Transactions on Vehicular Technology, vol. 67, no. 12, pp. 11354- 11364, Dec. 2018, doi: 10.1109/TVT.2018.2876788.

[34] K. Werfli et al., "Experimental Demonstration of High-Speed $4 \times 4$ Imaging Multi-CAP MIMO Visible Light Communications," in Journal of Lightwave Technology, vol. 36, no. 10, pp. 19441951, 15 May15, 2018, doi: 10.1109/JLT.2018.2796503.

[35] K. Reddy Sekhar and R. Mitra, "Performance analysis of DCO-OFDM over precoded massive MIMO VLC channel," 2018 IEEE International Conference on Advanced Networks and Telecommunications Systems (ANTS), 2018, pp. 1-6, doi: 10.1109/ANTS.2018.8710069.

[36] I. Tavakkolnia, C. Chen, R. Bian and H. Haas, "Energy-Efficient Adaptive MIMO-VLC Technique for Indoor LiFi Applications," 2018 25th International Conference on Telecommunications (ICT), 2018, pp. 331-335, doi: 10.1109/ICT.2018.8464933.

[37] N. V. Khanh, P. Q. Thai, N. H. Duy, and N. N. A. Khoa, "Investigation on MIMO OLED VLC System Performance," in Advanced Photonics 2018 (BGPP, IPR, NP, NOMA, Sensors, Networks, SPPCom, SOF), OSA Technical Digest (online) (Optical Society of America, 2018), paper JTu5A.61.

[38] A. Chaaban, Z. Rezki and M. Alouini, "MIMO intensity-modulation channels: Capacity bounds and high SNR characterization," 2017 IEEE International Conference on Communications (ICC), 2017, pp. 1-6, doi: 10.1109/ICC.2017.7997125.

[39] C. Chen, W. Zhong and D. Wu, "On the coverage of multiple-input multiple-output visible light communications [Invited]," in IEEE/OSA Journal of Optical Communications and Networking, vol. 9, no. 9, pp. D31-D41, Sept. 2017, doi: 10.1364/JOCN.9.000D31.

[40] A. Yesilkaya, E. Basar, F. Miramirkhani, E. Panayirci, M. Uysal and H. Haas, "Optical MIMOOFDM With Generalized LED Index Mod- ulation," in IEEE Transactions on Communications, vol. 65, no. 8, pp. 3429-3441, Aug. 2017, doi: 10.1109/TCOMM.2017.2699964.

[41] Y. Hong, L. Chen, and J. Zhao, "Experimental Demonstration of Performance-enhanced MIMOOFDM Visible Light Communications," in Optical Fiber Communication Conference, OSA Technical Digest (online) (Optical Society of America, 2017), paper Th1E.2.

[42] R. Sornalatha and N. Janakiraman, "Multiuser Visible Light Commu- nication System For Reliable Wireless Communication," 2017 IEEE International Conference on Computational Intelligence and Computing Research (ICCIC), 2017, pp. 1-6, doi: 10.1109/ICCIC.2017.8524159.

[43] Abhinav Johri, Farooq Husain and Ekta Agarwal, "A Review: Visible Light Communication using MU-MIMO-OFDM" SSRG International Journal of Electronics and Communication Engineering 4.4 (2017): 16-20. 
[44] X. Guo, Y. Guo and S. Li, "Adaptive transmission for MIMO VLC systems," 2017 IEEE/CIC International Conference on Communications in China (ICCC Workshops), 2017, pp. 1-4, doi: 10.1109/ICCChi- naW.2017.8355275.

[45] L. U. Khan, "Visible light communication: Applications, architecture, standardization and research challenges," Digit. Commun. Networks, vol. 3, no. 2, pp. 78-88, 2017.

[46] T. Q. Wang, C. He and J. Armstrong, "Performance Analysis of Aperture-Based Receivers for MIMO IM/DD Visible Light Communi- cations," in Journal of Lightwave Technology, vol. 35, no. 9, pp. 1513- 1523, 1 May1, 2017, doi: 10.1109/JLT.2016.2641002.

[47] O. Narmanlioglu, R. C. Kizilirmak, T. Baykas and M. Uysal, "Link Adaptation for MIMO OFDM Visible Light Communication Systems," in IEEE Access, vol. 5, pp. 26006-26014, 2017, doi: 10.1109/AC- CESS.2017.2771333.

[48] Zhao, Q., Fan, Y., and Kang, B., "A joint precoding scheme for indoor downlink multiuserMIMO VLC systems", $i_{i}$ Optics Communications $; / i_{i}$, vol. 403, pp. 341-346, 2017. doi:10.1016/j.optcom.2017.07.059.

[49] A. Yesilkaya, E. Basar, F. Miramirkhani, E. Panayirci, M. Uysal and H. Haas, "Optical MIMOOFDM With Generalized LED Index Mod- ulation," in IEEE Transactions on Communications, vol. 65, no. 8, pp. 3429-3441, Aug. 2017, doi: 10.1109/TCOMM.2017.2699964.

[50] C. Chen, W. Zhong and D. Wu, "Non-hermitian symmetry orthogo- nal frequency division multiplexing for multiple-input multiple-output visible light communications," in IEEE/OSA Journal of Optical Com- munications and Networking, vol. 9, no. 1, pp. 36-44, Jan. 2017, doi: 10.1364/JOCN.9.000036. 